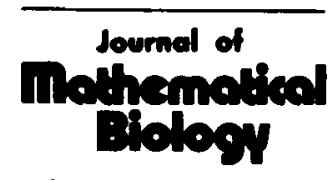

(C) by Springer-Verlag 1978

\title{
Bifurcation of Periodic Oscillations Due to Delays in Single Species Growth Models
}

\author{
J. M. Cushing \\ Department of Mathematics, Building 89, University of Arizona, Tucson, Arizona, 85721, USA
}

\begin{abstract}
Summary. Theorems are given which guarantee the bifurcation of non-constant, periodic solutions (of fixed period) of a scalar functional equation with two independent parameters. These results are applied to a single, isolated species growth model of general form with a general Volterra (Stieltjes) delay using the 'magnitudes' of the instantaneous and delayed growth rate responses as the independent bifurcation parameters. The case of linear growth rate responses (i.e. delay logistic models) is considered in more detail, particularly the often studied single lag logistic equation.
\end{abstract}

Key words: Growth models - Oscillations - Delays

\section{Introduction}

It is well known that a delay in the per unit growth rate response of a single, isolated species can cause the destabilization of an otherwise stable equilibrium density (see, for example $[5,12]$ ). The general effect of such a delay is to cause oscillations around the equilibrium. These oscillations can be either convergent, divergent or sustained depending on the 'significance' of the delay as compared to other parameters in the model (e.g. the inherent species growth rate). The latter possibility of sustained, periodic oscillations around an equilibrium has been studied mathematically by several authors, beginning with the early work of Wright [16], Kakutani and Markus [11], Jones [9, 10] and Grafton [8] on the singlelag logistic model and continuing with more recent work of Nussbaum [14]. Logistic models with more general delays have been studied by Dunkel $[6,7]$, Walther [15] and Cushing $[1,2,5]$.

Our purpose here is to demonstrate the existence of nonconstant periodic solutions of much more general single species models than those considered in these references and to do this in a new way.

The majority of the mathematical literature concerning nonconstant periodic solutions of functional equations utilizes the concept of a non-ejective fixed point (e.g. $[9,15])$, an approach which requires an extraordinary amount of detailed 
analysis of the special nature of the specific equations considered. Here we approach the problem of periodic solutions as a bifurcation phenomenon, but we do this in a manner slightly different from the classical Hopf technique which studies the problem as one involving one explicit 'bifurcation' parameter. We will assume that more than one independent parameter appears explicitly in the model. Mathematically, as can be seen by the shortness of the proof of our main result, Theorem 1 below, the advantage of this approach lies in the ease with which a bifurcation result is proved and in the generality of the models to which the result applies. The main conceptual difference between our approach and the classical Hopf bifurcation approach is that rather than treat the period as an unknown parameter we will consider bifurcation in a function space of fixed period by utilizing two parameters (rather than one) which appear explicitly in the equation. The appearance of two or more independent parameters in an equation is of course not unusual and is in fact highly likely in most applications. In the growth models considered below we will utilize three parameters: the inherent species growth rate and the 'magnitudes' of the delayed and nondelayed growth rate response to density changes.

In the following section we present our main mathematical results together with the necessary preliminaries. Application to single species growth models is made in Section 3. Although the main application is to general models with very general delay functionals, we also discuss the important special case of linear response functionals ('logistic' models) and in Section 4 the special case of a single-lag logistic model.

\section{The Main Theorem}

In preparation for our consideration of single species delay models in Section 3, we consider the scalar functional equation

$$
\text { (N) } x^{\prime}(t)=-\lambda_{1} x(t)-\lambda_{2} \int_{0}^{\infty} x(t-s) d h(s)+g\left(x, \lambda_{1}, \lambda_{2}\right)(t)
$$

where $\lambda_{1}, \lambda_{2}$ are reals and $\int_{0}^{\infty} d h(s)=1$. We will make specific assumptions about the integrator $h(s)$ and the functional $g$ below. The essential requirement will be that $g$ is 'higher order' in $x$ near $x=0$ and as a result we first study the linear problems

$$
y^{\prime}(t)=-\alpha_{1} y(t)-\alpha_{2} \int_{0}^{\infty} y(t-s) d h(s)
$$

(NH) $z^{\prime}(t)=-\alpha_{1} z(t)-\alpha_{2} \int_{0}^{\infty} z(t-s) d h(s)-f(t)$

where $f(t) \in P(p)$, the Banach space of continuous $p$-periodic functions under the supremum norm $|f|_{0}=\sup _{0 \leqslant t \leqslant p}|f(t)|$.

Assume $h(s)$ is of finite total variation on the half line $s \geqslant 0$ and $\int_{0}^{\infty} d h(s)=1$. 
The facts contained in the following lemma are known (a proof can be found in [4]). The period $p$ will always be assumed to be minimal.

Lemma 1. (A Fredholm Alternative.) Let $\alpha_{1}, \alpha_{2}$ and $p$ be fixed reals with $p>0$.

(a) The homogenous problem $(\mathrm{H})$ possesses at most a finite number $k \geqslant 0$ of independent, nontrivial p-periodic solutions $y_{i}(t) \in P(p)$.

(b) If $k=0$ then the nonhomogeneous equation $(\mathrm{NH})$ possesses a unique solution $z(t) \in P(p)$ for every $f(t) \in P(p)$.

(c) If $k>0$ then $(\mathrm{NH})$ has a solution $z(t) \in P(p)$ if and only if $f(t) \in P_{0}(p)$ where $P_{0}(p)$ is the Banach space

$$
P_{0}(p)=\left\{f \in P(p):\left(f, y_{i}\right):=p^{-1} \int_{0}^{p} f(t) y_{i}(t) d t=0, \quad 1 \leqslant i \leqslant k\right\} .
$$

If $k>0$ and $f(t) \in P_{0}(p)$ then $(\mathrm{NH})$ has a unique solution $z(t) \in P_{0}(p)$ and the operator $L: P_{0}(p) \rightarrow P_{0}(p)$ defined by $z=L f$ is linear and compact.

By a solution we always mean a function which is differentiable almost everywhere.

We are interested in those values of $\alpha_{i}$ for which the homogeneous equation $(\mathrm{H})$ possesses exactly two nontrivial, $p$-periodic solutions. Let

$$
S_{n}(p):=\int_{0}^{\infty} \sin 2 n \pi p^{-1} s d h(s), \quad C_{n}(p):=\int_{0}^{\infty} \cos 2 n \pi p^{-1} s d h(s) .
$$

Lemma 2. The homogeneous problem $(\mathrm{H})$ possesses exactly two independent, nonconstant p-periodic solutions if and only if $p>0$ is such that the two conditions

$$
\text { (H1) } S_{1}(p) \neq 0, \quad C_{1}(p) \neq 1
$$

and

(H2) for each integer $n>1$ either $S_{n}(p) \neq n S_{1}(p)$ or $C_{1}(p) \neq C_{n}(p)$

hold and also the coefficients are given by

$$
\alpha_{1}=-2 \pi C_{1}(p) / p S_{1}(p), \quad \alpha_{2}=2 \pi / p S_{1}(p) .
$$

The nontrivial solutions are then $y_{1}(t)=\sin 2 \pi p^{-1} t, y_{2}(t)=\cos 2 \pi p^{-1} t$.

Proof. If the complex Fourier series

$$
y(t)=\sum_{n=-\infty}^{+\infty} c_{n} e^{2 \pi i n p^{-1} t}
$$

where $c_{n}=\bar{c}_{-n}, n>0,\left(^{(-)}\right.$denotes complex conjugation) is substituted into (H) and coefficients of like terms are equated, then one obtains the equations

$$
\left[\alpha_{1}+\alpha_{2} C_{n}(p)+i\left(2 \pi n p^{-1}-\alpha_{2} S_{n}(p)\right)\right] c_{n}=0, \quad n \geqslant 0 .
$$

Since nontrivial solutions are obtained if and only if at least one coefficient $c_{n} \neq 0$; since each nonzero coefficient $c_{n}$ for $n>0$ yields two independent solutions (namely, $\sin 2 \pi n p^{-1} t$ and $\cos 2 \pi n p^{-1} t$ ); and since $p$ is assumed minimal we see that $(\mathrm{H})$ has 
exactly two independent $p$-periodic solutions $\sin 2 \pi p^{-1} t$ and $\cos 2 \pi p^{-1} t$ if and only if

$$
\begin{array}{lll}
\alpha_{1}+\alpha_{2} \neq 0 & & (n=0) \\
\alpha_{1}+\alpha_{2} C_{1}(p)=0, & \alpha_{2} S_{1}(p)=2 \pi p^{-1} & (n=1) \\
\alpha_{1}+\alpha_{2} C_{n}(p) \neq 0 & \text { or } \quad \alpha_{2} S_{n}(p) \neq 2 \pi n p^{-1} & (n \geqslant 2) .
\end{array}
$$

The second pair of equations yield the values of $\alpha_{1}$ and $\alpha_{2}$ as given in the lemma (provided $S_{1}(p) \neq 0$ ) while the remaining conditions are easily seen to be equivalent to $(\mathrm{H} 1)$ and (H2).

Example 1. Suppose $h(s)=u_{T}(s)$, the unit step function at $t=T$, so that the delay in equations $(\mathrm{H})$ and $(\mathrm{NH})$ reduces to a constant, instantaneous time lag of length $T>0$. Then (H1) reduces to $\sin 2 \pi p^{-1} T \neq 0$. Moreover, it is easy to show that (H2) is fulfilled, for if both $\sin 2 \pi n p^{-1} T=n \sin 2 \pi p^{-1} T$ and $\cos 2 \pi p^{-1} T=\cos 2 n \pi p^{-1} T$ hold for some integer $n \geqslant 2$ then the contradiction $\left(n^{2}-1\right) \sin ^{2} 2 \pi p^{-1} T=0$ follows. For this example, $\alpha_{1}=-2 \pi p^{-1} \cot 2 \pi p^{-1} T, \alpha_{2}=2 \pi p^{-1} \csc 2 \pi p^{-1} T$.

Example 2. An example for the case of continuously distributed delays is $d h(s)=$ $k(s) d s$ where

$$
k_{m}(s)= \begin{cases}w^{2} s e^{-w s} & \text { for } m=1 \\ w e^{-w s} & \text { for } m=0\end{cases}
$$

and $w>0$ is a positive real. Note that $k_{m}(s) \geqslant 0$ and $\int_{0}^{\infty} k_{m}(s) d s=1$. Such kernels are often used in delay models (e.g. see $[12,5])$. The kernel $k_{0}(t)$ represents a 'weak' delay in the sense that it is monotonically decreasing while the kernel $k_{1}(s)$ represents a 'strong' delay in the sense that it increases to a maximum before monotonically decreasing to zero. Thus $k_{1}(s)$ provides a continuously distributed analogue to the case of a single, instantaneous time lag.

For these kernels we have (setting $f=2 \pi p^{-1}$ )

$$
\begin{aligned}
& S_{n}^{(m)}(p):=\int_{0}^{\infty} k_{m}(s) \sin n f s d s= \begin{cases}2 n f w^{3}\left(w^{2}+n^{2} f^{2}\right)^{-2}, & m=1 \\
n w f\left(w^{2}+n^{2} f^{2}\right)^{-1}, & m=0\end{cases} \\
& C_{n}^{(m)}(p):=\int_{0}^{\infty} k_{m}(s) \cos n f s d s= \begin{cases}w^{2}\left(w^{2}-n^{2} f^{2}\right)\left(w^{2}+n^{2} f^{2}\right)^{-2}, & m=1 \\
w^{2}\left(w^{2}+n^{2} f^{2}\right)^{-1}, & m=0 .\end{cases}
\end{aligned}
$$

Since $S_{n}^{(m)}(p)>0, C_{n}^{(m)}(p)<1$ for all $n, m, w$ and $f$ we see that (H1) holds. It is easy to see that $n S_{1}^{(m)}(p) \neq S_{n}^{(m)}(p), n>1$, so that (H2) holds also. For these delay kernels we find that

$$
\begin{aligned}
& \alpha_{1}= \begin{cases}\left(f^{2}-w^{2}\right) / 2 w, & m=1 \\
-w, & m=0\end{cases} \\
& \alpha_{2}= \begin{cases}\left(w^{2}+f^{2}\right)^{2} / 2 w^{3}, & m=1 \\
\left(w^{2}+f^{2}\right) / w, & m=0 .\end{cases}
\end{aligned}
$$


With these preliminaries concerning the linear problems $(\mathrm{H})$ and $(\mathrm{NH})$ out of the way, we return to the nonlinear problem $(\mathrm{N})$. Let $B_{p}(r)$ denote the ball $B_{p}(r)=$ $\left\{x \in P(p):|x|_{0} \leqslant r\right\}$ of radius $r$ in $P(p)$ and let $B_{p}^{0}(r)$ denote the corresponding ball in $P_{0}(p)$. Let $I\left(\alpha_{i}, r\right)=\left(\alpha_{i}-r, \alpha_{i}+r\right)$ and $I(r)=I\left(\alpha_{1}, r\right) \times I\left(\alpha_{2}, r\right)$. We will need the following hypotheses on the higher order terms in $(\mathrm{N})$.

(H3): Assume (H1) and (H2) hold for some $p>0$. Suppose that the operator $g: B_{p}(r) \times I(r) \rightarrow P(p)$ is continuous for some $r>0$, that for each pair $\left(\lambda_{1}, \lambda_{2}\right) \in$ $I(r)$ it maps bounded sets in $B_{p}(r)$ into bounded sets and that $\left|g\left(x, \lambda_{1}, \lambda_{2}\right)\right|_{0}=$ $o\left(|x|_{0}\right)$ near $x=0$ uniformly for $\left(\lambda_{1}, \lambda_{2}\right) \in I(r)$. Finally, for each $x \in B_{p}(r)$ suppose that $g$ is (Fréchet) continuously differentiable in both $\lambda_{i} \in I\left(\alpha_{i}, r\right)$ with $\left|\left(\partial / \partial \lambda_{i}\right) g\left(x, \lambda_{1}, \lambda_{2}\right)\right|_{0}=o\left(|x|_{0}\right)$ near $x=0$ uniformly in $\left(\lambda_{1}, \lambda_{2}\right) \in I(r)$.

In our applications below $g$ will be linear in $\lambda_{i}$. Such an operator $g=\lambda_{1} g_{1}(x)+$ $\lambda_{2} g_{2}(x)+g_{3}(x)$ will satisfy (H3) when each operator $g_{i}(x)$ satisfies those conditions in (H3) involving $g$ as an operator in $x$.

Theorem 1. Assume that (H1), (H2) and (H3) hold for some period $p>0$ and some delay integrator $h(s)$ of finite total variation on $s \geqslant 0, \int_{0}^{\infty} d h(s)=1$. Let $y=k_{1} \sin 2 \pi p^{-1} t+k_{2} \cos 2 \pi p^{-1} t, k_{1}^{2}+k_{2}^{2}=1$, be any nontrivial solution of $(\mathrm{H})$ for the $\alpha_{i}$ given in Lemma 2. For each small $|\varepsilon|$ there exists a solution of $(\mathrm{N})$ of the form

$$
x(t)=\varepsilon y(t)+\varepsilon z(t, \varepsilon), \quad z(., \varepsilon) \in P_{0}(p), \quad|z(., \varepsilon)|_{0}=0(|\varepsilon|)
$$

for $\lambda_{i}=\alpha_{i}+\beta_{i}(\varepsilon)$ where $\left|\beta_{i}(\varepsilon)\right|=0(|\varepsilon|)$.

Proof. Substitution of (2.1) and $\lambda_{i}$ into (N) yields, after a cancellation of $\varepsilon$, the equation

$$
z^{\prime}(t)=-\alpha_{1} z(t)-\alpha_{2} \int_{0}^{\infty} z(t-s) d h(s)-G\left(z, \beta_{i}, \varepsilon\right)
$$

where

$$
\begin{aligned}
G\left(z, \beta_{i}, \varepsilon\right):= & \beta_{1}(y+z)+\beta_{2} \int_{0}^{\infty}(y(t-s)+z(t-s) d h(s) \\
& -\varepsilon^{-1} g\left(\varepsilon y+\varepsilon z, \alpha_{1}+\beta_{1}, \alpha_{2}+\beta_{2}\right)
\end{aligned}
$$

which must be solved for $z \in P_{0}(p)$ for appropriate $\beta_{i}$. In as much as $G \in P(p)$ it follows from Lemma 1 (c) that in order for $z \in P_{0}(p)$ it is necessary that $G \in P_{0}(p)$. To solve (2.2) we will prove the existence of a fixed point of an operator defined as follows: given any $Z \in B_{p}^{0}(r)$ we choose the constants $\beta_{i}$ such that $G\left(Z, \beta_{i}, \varepsilon\right)$ lies in $P_{0}(p)$ in which case $z=L G\left(Z, \beta_{i}, \varepsilon\right)$ is the unique solution in $P_{0}(p)$ of the linear nonhomogeneous equation (NH) with $f(t)=G\left(Z, \beta_{i}, \varepsilon\right)$. Clearly a fixed point of this operator solves the nonlinear equation (2.2).

In order to accomplish this task we must first show that the $\beta_{i}$ can be chosen so that $G\left(z, \beta_{i}, \varepsilon\right) \in P_{0}(p)$, a requirement which yields the following two equations for $\beta_{1}, \beta_{2}$ :

$$
a_{i 1}(y+z) \beta_{1}+a_{i 2}(y+z) \beta_{2}-\gamma_{i}\left(y+z, \beta_{1}, \beta_{2}, \varepsilon\right)=0, \quad i=1 \text { and } 2
$$


where

$$
\begin{aligned}
& a_{11}(x)=\left(x(t), \sin 2 \pi p^{-1} t\right), \quad a_{21}(x)=\left(x(t), \cos 2 \pi p^{-1} t\right) \\
& a_{12}(x)=\left(\int_{0}^{\infty} x(t-s) d h(s), \sin 2 \pi p^{-1} t\right), \\
& a_{22}(x)=\left(\int_{0}^{\infty} x(t-s) d h(s), \cos 2 \pi p^{-1} t\right) \\
& \gamma_{1}\left(x, \beta_{1}, \beta_{2}, \varepsilon\right)=\left(\varepsilon^{-1} g\left(\varepsilon x, \alpha_{1}+\beta_{1}, \alpha_{2}+\beta_{2}\right), \sin 2 \pi p^{-1} t\right) \\
& \gamma_{2}\left(x, \beta_{1}, \beta_{2}, \varepsilon\right)=\left(\varepsilon^{-1} g\left(\varepsilon x, \alpha_{1}+\beta_{1}, \alpha_{2}+\beta_{2}\right), \cos 2 \pi p^{-1} t\right)
\end{aligned}
$$

for $x \in P(p)$ and $\varepsilon$ small enough. Equations (2.3) constitute two equations for two unknown $\beta_{i}$. The Jacobian $J=J(z, \varepsilon)$ with respect to $\beta_{1}, \beta_{2}$ of the left hand side of (2.3) is, for $z=0$ and $\varepsilon=0$, equal to $J(0,0)=\left(a_{i j}(y)\right)$. A simple calculation shows that $\operatorname{det}\left(a_{i j}(y)\right)=-S_{1}(p) / 4$ and consequently det $J(0,0) \neq 0$ by (H1). The implicit function theorem implies that (2.3) is uniquely solvable for $\beta_{i}=\beta_{i}(z, \varepsilon)$ where $\beta_{i}(0,0)=0$ and $\beta_{i}$ is continuous in $z$ and $\varepsilon$ for $z \in B_{p}^{0}\left(r^{*}\right)$ and $|\varepsilon| \leqslant \varepsilon^{0}$, $0<r^{*} \leqslant r, \varepsilon^{0}>0$. This implies $\beta_{i}$ has a bounded range (for $r^{*}$ and $\varepsilon^{0}$ small) so that the hypotheses on $g$ in (H3) imply $\left|\gamma_{i}\right|=0(|\varepsilon|)$ uniformly for $z \in B_{p}^{0}\left(r^{*}\right)$. This fact, together with the fact that $\left(a_{i j}(y+z)\right)=J(z, \varepsilon)+O(|\varepsilon|)$ uniformly for $z \in B_{p}^{0}\left(r^{*}\right)$, implies from (2.3) that $\left|\beta_{i}\right|=0(|\varepsilon|)$ uniformly for $z \in B_{p}^{0}\left(r^{*}\right)$.

The operator $Q_{\varepsilon}: B_{p}^{0}\left(r^{*}\right) \rightarrow P_{0}(p)$ is thus well defined by $Q_{\varepsilon} z=L G\left(z, \beta_{i}(z, \varepsilon), \varepsilon\right)$ and is continuous for each $\varepsilon,|\varepsilon| \leqslant \varepsilon^{0}$. Since $L$ is compact, $Q_{\varepsilon}$ is completely continuous. The hypotheses on $g$ in $(\mathrm{H} 3)$ and the properties of $\beta_{i}$ imply $\left|Q_{\varepsilon} z\right|_{0}=$ $O(|\varepsilon|)$ uniformly for $z \in B_{p}^{0}\left(r^{*}\right)$ and consequently for $\varepsilon^{0}$ smaller, if necessary, $Q_{\varepsilon}$ maps $B_{p}^{0}\left(r^{*}\right)$ into itself for each $|\varepsilon| \leqslant \varepsilon^{0}$. The Schauder-Tychonoff fixed point theorem guarantees the existence of a solution of (2.2) in $B_{p}^{0}\left(r^{*}\right)$ for each $|\varepsilon| \leqslant \varepsilon^{0}$. Since $z=Q_{\varepsilon} z$ it follows that $|z|_{0}=0(|\varepsilon|)$.

Remark 1. The Schauder-Tychonoff fixed point theorem was used in the above proof. If a suitable Lipschitz-type assumption is made on the functional $g$, then the contraction principle could be used with the added result that the fixed point is unique in $B_{p}^{0}\left(r^{*}\right)$. In this case the small amplitude solutions found in Theorem 1 would be, for a given $p$, the only small amplitude solutions.

It is often of interest in applications to be able to compute some higher order terms in the $\beta_{i}$.

This would be the case, for example, if the 'direction of bifurcation' were desired; that is to say, if it is desired to know whether $\lambda_{i} \geqslant \alpha_{i}$ or $\lambda_{i} \leqslant \alpha_{i}$ (or neither) for small $|\varepsilon|$. The familiar classical method for doing this is to substitute $\varepsilon$ series expansions for $x$ and $\lambda_{i}$ into $(\mathrm{N})$ and to equate coefficients of like powers of $\varepsilon$. This generates a recursive sequence of linear equations, the first of which is $(H)$ and the remaining of which have the nonhomogeneous form $(\mathrm{NH})$. The orthogonality conditions of Lemma 1(c) for these latter equations determine the successivi coefficients in the $\varepsilon$ expansion of $\lambda_{i}$. This procedure is illustrated in Section 4 for the lag-logistic equation. We complete this section with a formal justification of this method. We will need stronger hypotheses on the nonlinear term $g$ in $(N)$ : 
(H4): Assume (H1) and (H2) holds for some $p>0$. Suppose that $m \geqslant 2$ is an integer, $y_{j} \in P(p)$ are $m$ arbitrary but fixed functions $(1 \leqslant j \leqslant m)$ and $\alpha_{j, i}$ are $2 m-2$ arbitrary but fixed constants $(2 \leqslant j \leqslant m, 1 \leqslant i \leqslant 2)$. Assume that there exists $r>0$ and $\varepsilon^{0}>0$ such that for any $z \in B_{p}(r),\left|\beta_{i}\right| \leqslant r$ and $|\varepsilon| \leqslant \varepsilon^{0}$ if

$$
\begin{aligned}
& x=\sum_{j=1}^{m} \varepsilon^{j} y_{j}+\varepsilon^{m} z \\
& \lambda_{i}=\sum_{j=1}^{m} \alpha_{j, i} \varepsilon^{j-1}+\varepsilon^{m-1} \beta_{i}, \quad \alpha_{1, i}=\alpha_{i}
\end{aligned}
$$

then

$$
g\left(x, \lambda_{1}, \lambda_{2}\right)=\sum_{j=2}^{m} \varepsilon^{j} g_{j}+\varepsilon^{m} g_{m+1}
$$

where $g_{j}=g_{j}\left(y_{q}, \alpha_{q, i}\right) \in P(p)$ for $1 \leqslant q \leqslant j-1,2 \leqslant j \leqslant m$ and where $g_{m+1}$ is continuous in $z, \beta_{i}$ and $\varepsilon$ and (Fréchet) continuously differentiable with respect to $\beta_{i}$ with $\left|g_{m+1}\right|_{0}=0(|\varepsilon|)$ and $\left|\left(\partial / \partial \beta_{i}\right) g_{m+1}\right|_{0}=0(|\varepsilon|)$ uniformly in $z$ and $\beta_{i}$.

This hypothesis (H4) says essentially that when $m$ th order (respectively $(m-1)$ st order) expansions in $\varepsilon$ for $x$ (respectively $\lambda_{i}$ ) are substituted into the operator $g$ there results an expansion in $\varepsilon$ whose coefficients depend only on coefficients in $x$ and $\lambda_{i}$ of equal or less order. It holds if $g$ is $m$ times continuously (Fréchet) differentiable in $x$ and $\lambda_{i}$.

As an illustration of $(\mathrm{H} 4)$ (which is pertinent to our application below) we remark that it is satisfied by operators of the form

$$
g\left(x ; \lambda_{1}, \lambda_{2}\right)=\lambda_{1} g_{1}(x)+\lambda_{2} g_{2}\left(\int_{0}^{\infty} x(t-s) d h(s)\right)
$$

where $g_{i}$ are $m$ times continuously differentiable real valued functions of real variables for which $g_{i}(0)=g_{i}^{\prime}(0)=0$.

We now define what will turn out to be the coefficients in the expansions of solutions $x$ and $\lambda_{i}$ of Equation $(\mathrm{N})$. Let $y_{1}(t)=y(t)$ be any nontrivial $p$-periodic solution of $(\mathrm{H})$ and $\alpha_{1, i}=\alpha_{i}$ (from Lemma 2). Define $y_{j}$ and $\alpha_{j, i}$ for $2 \leqslant j \leqslant m$ and $i=1,2$, recursively as follows:

$$
\begin{aligned}
& y_{j}^{\prime}=-\alpha_{1} y_{j}-\alpha_{2} \int_{0}^{\infty} y_{j}(t-s) d h(s)+G_{j}(t), \quad y_{j} \in P_{0}(p) \\
& G_{j}:=-\sum_{q=1}^{j-1}\left(\alpha_{j+1-q, 1} y_{q}+\alpha_{j+1-q, 2} \int_{0}^{\infty} y_{q}(t-s) d h(s)\right)+g_{j}, \quad 2 \leqslant j \leqslant m \\
& a_{i 1}(y) \alpha_{j, 1}+a_{t 2}(y) \alpha_{j, 2}+\gamma_{j, i}=0, \quad 2 \leqslant j \leqslant m, \quad 1 \leqslant i \leqslant 2
\end{aligned}
$$

where $a_{i j}(y)$ are defined as above in the proof of Theorem 1 and

$$
\begin{aligned}
& \gamma_{j, 1}:=\left(H_{j}(t), \sin 2 \pi p^{-1} t\right), \quad \gamma_{j .2}:=\left(H_{j}(t), \cos 2 \pi p^{-1} t\right) . \\
& H_{j}:=\sum_{q=2}^{j-1}\left(\alpha_{j+1-q, 1} y_{q}+\alpha_{j+1-q, 2} \int_{0}^{\infty} y_{q}(t-s) d h(s)\right)-g_{j}, \quad 2 \leqslant j \leqslant m .
\end{aligned}
$$


The $2 \times 2$ linear system for $\alpha_{j, 1}$ and $\alpha_{j, 2}$ given by (2.5), which is uniquely solvable because its coefficient matrix has determinant $-S_{2}(p) / 4$ as in the proof of Theorem 1 , is equivalent to $G_{j} \in P_{0}(p)$ and hence allows (2.4) to be uniquely solved in $P_{0}(p)$. Note that the properties of $g_{j}$ given in (H4) allow (2.4) and (2.5) to be solved recursively since $G_{f}$ and $H_{j}$ depend only on previously calculated $y_{q}$ and $\alpha_{q, i}, 2 \leqslant$ $q \leqslant j-1$.

Theorem 2. Assume (H1), (H2) and (H4) hold for some $p>0$ and integer $m \geqslant 2$. Let $y_{j}(t)$ and $\alpha_{j, i}$ for $1 \leqslant j \leqslant m, 1 \leqslant i \leqslant 2$ be defined by (2.4) and (2.5). For small $|\varepsilon|$ there exists a solution of $(\mathrm{N})$ of the form

$$
x(t)=\sum_{j=1}^{m} \varepsilon^{j} y_{j}(t)+\varepsilon^{m} z(t, \varepsilon)
$$

with

$$
\lambda_{i}=\alpha_{i}+\sum_{j=2}^{m} \varepsilon^{j-1} \alpha_{j, i}+\varepsilon^{m-1} \beta_{i}(\varepsilon), \quad 1 \leqslant i \leqslant 2,
$$

where

$$
z(\cdot, \varepsilon) \in P_{0}(p), \quad|z|_{0}=0(|\varepsilon|)
$$

and

$$
\left|\beta_{i}(\varepsilon)\right|=0(|\varepsilon|) .
$$

Proof. Substitution of the expansions (2.6) and (2.7) into $(\mathrm{N})$ yields, because of equations (2.4) and (2.5) and after a cancellation of an $\varepsilon^{m}$, an equation of the form

$$
\begin{aligned}
z^{\prime}= & -\alpha_{1} z-\alpha_{2} \int_{0}^{\infty} z(t-s) d h(s)-\beta_{1}\left(\sum_{j=1}^{m} \varepsilon^{j-1} y_{j}+\varepsilon^{m-1} z\right) \\
& -\beta_{2}\left(\sum_{j=1}^{m} \varepsilon^{j-1} \int_{0}^{\infty} y_{j}(t-s) d h(s)+\varepsilon^{m-1} \int_{0}^{\infty} z(t-s) d h(s)\right) \\
& +R\left(z, \beta_{i}, \varepsilon\right)+p(t, \varepsilon)
\end{aligned}
$$

to be solved for $z \in B_{p}^{0}(r)$ for appropriate $\beta_{i}$.

Here $p(\cdot, \varepsilon) \in P_{0}(p)$ satisfies $|p|_{0}=0(|\varepsilon|)$ and by (H4) both $|R|_{0}$ and $\left|\left(\partial / \partial \beta_{i}\right) R\right|_{0}=$ $O(|\varepsilon|)$ uniformly for $z \in B_{p}^{0}(r)$ and $\left|\beta_{i}\right| \leqslant r$. This Equation (2.8) can be shown to have a solution in $B_{p}^{0}(r)$ in exactly the same way that (2.2) was: the necessary condition that the last four terms on the right hand side of $(2.8)$ lie in $P_{0}(p)$ leads to two equations for the two constants $\beta_{i}$ which are solvable by the implicit function theorem (the Jacobian at $z=0, \varepsilon=0$, is the same as that in the proof of Theorem 1) for $\beta_{i}=\beta_{i}(z, \varepsilon)$ which have the same properties as in the proof of Theorem 1 . The operator $Q_{\varepsilon}$ has the same properties as the analogous operator in the proof of Theorem 1 and the proof follows exactly as in the last paragraph of that proof. 


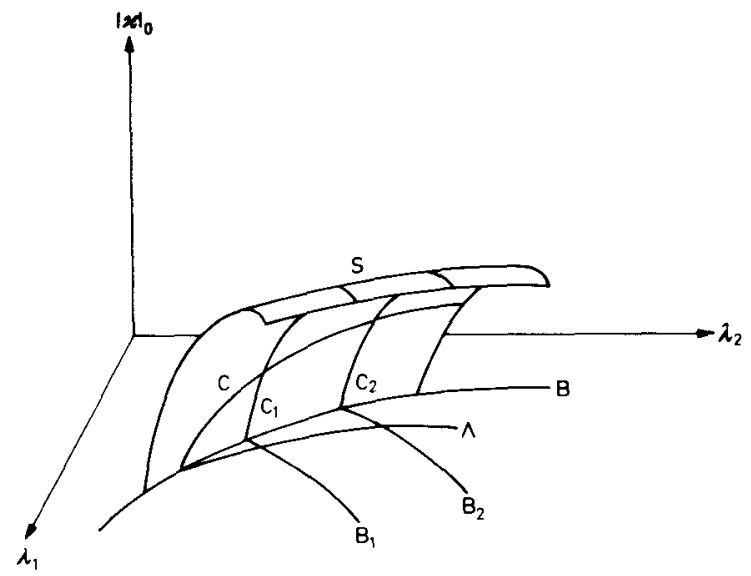

Figure 1

The result of Theorem 1 is different from classical Hopf bifurcation in that bifurcation occurs within a space of fixed period with respect to two independent bifurcation parameters $\lambda_{1}, \lambda_{2}$. Hopf bifurcation deals with equations with one bifurcation parameter and consequently results (in general) in bifurcating solutions of varying period. The reason for this is that in order to deal with the two orthogonality conditions which result from the two independent solutions of the linearization, the Hopf approach must introduce a second parameter into the analysis, which it does by considering the unknown period of the solution. Our approach on the other hand uses two parameters which already appear explicitly in the equation, an approach which results in a short proof which applies in a general setting.

Viewed in a certain way these two different approaches can be seen to yield the same bifurcation phenomena. If Hopf bifurcation is seen as prescribing a relationship $\Lambda\left(\lambda_{1}, \lambda_{2}\right)=0$ between $\lambda_{1}$ and $\lambda_{2}$ so that there appears only one independent parameter, then in principle the equation $\Lambda\left(\lambda_{1}(\varepsilon, p), \lambda_{2}(\varepsilon, p)\right)=0$ (we now view $\lambda_{i}$ in Theorem 1 as functions of $p$ as well as $\varepsilon$ ) determines $p=p(\varepsilon)$ and hence the varying period in the bifurcating solutions of Theorem 1. Geometrically this comparison can be viewed as in Figure 1 where the amplitude of the solutions $x$ is plotted against the corresponding $\lambda_{1}, \lambda_{2}$. For fixed period $p=p_{j}$ Theorem 1 yields a branch curve $C_{j}$ above the base curve $B_{j}$ in the $\lambda_{1}, \lambda_{2}$ plane determined by $\lambda_{i}=\lambda_{i}\left(\varepsilon, p_{j}\right)$ as a function of $\varepsilon$. These curves $C_{j}$ and $B_{j}$ meet at the bifurcation point corresponding to $\varepsilon=0$ lying on the 'bifurcation curve' $B$ given by $\lambda_{i}=$ $\lambda_{i}(0, p)$ as a function of the period $p$. Thus, we obtain a 'bifurcation surface' $S$ swept out by the curves $C_{j}$ as the period varies. Now, if a relationship $\Lambda\left(\lambda_{1}, \lambda_{2}\right)=0$ is prescribed as a curve $\Lambda$ in the $\lambda_{1}, \lambda_{2}$ plane, then one obtains a bifurcation curve $C$ on $S$ with base curve $\Lambda$ which in general will be transversal to $C_{j}$ and hence will yield period varying, Hopf-type bifurcation. 
A figure similar to Figure 1 is drawn for the specific case of the lag logistic equation in Figure 2.

\section{Single Species Delay Models}

Suppose that the per unit growth rate of a single, isolated species of density $N=N(t)$ is modeled by an equation

$$
\frac{1}{N(t)} \frac{d N(t)}{d t}=b-a f(N(t))-d F(N)(t)
$$

where the term $f$ accounts for the instantaneous effect of species density on its growth rate while the functional expression $F$ accounts for any such delayed effects. The symbols $a, b$ and $d$ are parameters which, roughly speaking, can be thought of as follows: $a$ is a measure of the magnitude of the instantaneous density effects on growth rate, $b$ is the inherent growth rate of the species in the absence of any density effects (i.e. in an environment with unlimited resources) and finally $d$ is the magnitude of the delayed density effects on growth rate. We assume (3.1) has a positive equilibrium $N=e$ :

$\left(\mathrm{h}_{1}\right)$ the equation $a f(e)+d F(e)(t)=b$ has at least one (not necessarily unique) constant solution $e>0$.

We are interested in the existence of nonconstant, periodic solutions of (3.1) near $N=e$ for critical values of the parameters $a, b$, and $d$. To apply the results of the previous section we first center the problem on the equilibrium by setting $x=N-e$. If we assume that the functions $f$ and $F$ satisfy the following hypotheses:

$\left(\mathrm{h}_{2}\right)$ (a) $f$ is a real valued function of a real variable which is twice continuously differentiable in a neighbourhood of $N=e$ with $f^{\prime}(e) \neq 0$;

(b) $h(s)$ is an integrator of finite total variation on the half-line $s \geqslant 0$, $\int_{0}^{\infty} d h(s)=1$, and satisfies $(\mathrm{H} 1)$ and $(\mathrm{H} 2)$ for some period $p>0$ for which we can write

$$
F(x+e)(t)=F(e)+\int_{0}^{\infty} x(t-s) d h(s)+R(x)(t), \quad x \in B_{p}(r)
$$

for some $r>0$ where the higher order term $R(x)$ satisfies the conditions which $g$ satisfies in (H3),

then $x$ will satisfy an equation of the form (N) in Section 2 with

$$
\begin{aligned}
& \lambda_{1}=a e f^{\prime}(e), \quad \lambda_{2}=d e \\
& g\left(x, \lambda_{1}, \lambda_{2}\right):=\lambda_{1} g_{1}(x)+\lambda_{2} g_{2}(x) \\
& g_{1}(x):=-\left(e q(x)+f^{\prime}(e) x^{2}+x q(x)\right) / e f^{\prime}(e) \\
& g_{2}(x):=-\left(e R(x)+x \int_{0}^{\infty} x(t-s) d h(s)+x R(x)\right) / e \\
& q(x):=f(x+e)-f(e)-f^{\prime}(e) x .
\end{aligned}
$$


Moreover, under these conditions the resulting equation satisfies the hypotheses (H1), (H2) and (H3) of Section 2. We now view $a$ and $d$ as free parameters with $e$ fixed and $b$ determined by $\left(h_{1}\right)$. From Theorem 1 we obtain the following result.

Theorem 3. Assume that the response functions $f$ and $F$ in the single species model (3.1) satisfy hypotheses $\left(\mathrm{h}_{1}\right)$ and $\left(\mathrm{h}_{2}\right)$. For a fixed value of the equilibrium e the delay single species model (3.1) has nontrivial p-periodic solutions ( $p$ as in $(\mathrm{H} 1)$ and $(\mathrm{H} 2)$ ) of the form

$$
N(t)=e+\varepsilon y(t)+\varepsilon z(t, \varepsilon)
$$

for $|\varepsilon|$ small, $z(\cdot, \varepsilon) \in P_{0}(p)$ and for $y$ as in Theorem 1 with parameters

$$
\begin{aligned}
& a=\left(\alpha_{1}+\beta_{1}(\varepsilon)\right) / e f^{\prime}(e)=\alpha_{1} / e f^{\prime}(e)+O(\varepsilon) \\
& d=\left(\alpha_{2}+\beta_{2}(\varepsilon)\right) / e=\alpha_{2} / e+0(\varepsilon) \\
& b=a f(e)+d F(e)=\alpha_{1} f(e) / e f^{\prime}(e)+\alpha_{2} F(e) / e+O(\varepsilon),
\end{aligned}
$$

where the critical values $\alpha_{1}, \alpha_{2}$ are given in Lemma 2 and where $\beta_{i}=0(|\varepsilon|)$ and $|z(t, \varepsilon)|_{0}=0(|\varepsilon|)$.

As an illustration of Theorem 3 we consider the case when both $f$ and $F$ are linear:

$$
\frac{1}{N(t)} \frac{d N(t)}{d t}=b-a N(t)-d \int_{0}^{\infty} N(t-s) d h(s), \quad a+d \neq 0 .
$$

Here $\left(\mathrm{h}_{1}\right)$ holds and $e=b /(a+d)$, which we assume is positive. For convenience we will assume that the units used for species density are chosen so that $e=1$ or, in other words, we assume

$$
b=a+d .
$$

In this application $f(1)=1, f^{\prime}(1)=1, q(x)=0, R(x)=0$ and $F(1)=1$. All of the needed hypotheses on $f(N)=N$ and $F(N)=\int_{0}^{\infty} N(t-s) d h(s)$ hold. Thus all that is needed in order to apply Theorem 3 to (3.2) are the requirements that the period $p$ be chosen so that $(\mathrm{H} 1)$ and $(\mathrm{H} 2)$ hold for the given delay integrator $h(s)$. Thus we have the following corollary of Theorem 3 .

Corollary 1. If $p$ is chosen so that $(\mathrm{H} 1)$ and $(\mathrm{H} 2)$ hold then the linear-response model (3.2)-(3.3) has nontrivial p-periodic solutions of the form described in Theorem 2 (with $e=1$ ) for parameter values

$$
\begin{aligned}
& a=-2 \pi C_{1}(p) / p S_{1}(p)+0(\varepsilon), \quad d=2 \pi / p S_{1}(p)+0(\varepsilon) \\
& b=2 \pi\left(1-C_{1}(p)\right) / p S_{1}(p)+0(\varepsilon) .
\end{aligned}
$$

Remark 2. If $d h(s)=k(s) d s, k(s) \geqslant 0, \int_{0}^{\infty} k(s) d s=1$ then it is known [13] that $a>|d|$ implies the global asymptotic stability of the equilibrium of the delay logistic model (3.2). The result in Corollary 1 is consistent which this known fact since $\left|C_{1}(p)\right|<1$ implies, at least to lowest order in $\varepsilon$, that $|a|<|d|$. In this sense the growth rate response delay in (3.2) must be of sufficient magnitude in order that that the species density exhibit sustained oscillations. 
Remark 3. Remark 1 is applicable to the results in Theorem 3 and hence in Corollary 1. Thus, for the linear response model (3.2), the small amplitude solutions in Corollary 1 are unique.

Remark 4. In most linear-response models (3.2) the constants are to satisfy the sign conditions $b>0, a \geqslant 0, d \geqslant 0$. In applying Corollary 1 in this case we need only require, in addition (H2), the inequalities

$$
S_{1}(p)>0, \quad C_{1}(p)<0
$$

in order to insure these sign conditions (at least for small $\varepsilon$ ).

In the case of the instantaneous, constant time lag $h(s)=u_{T}(s)$ in Example 1 above, the sign requirements (3.4) reduce to

$$
\sin 2 \pi p^{-1} T>0, \quad \cos 2 \pi p^{-1} T<0 .
$$

The condition (H2) is fulfilled as was shown in Example 1 and consequently Corollary 1 guarantees the existence of nonconstant $p$-periodic solutions for the given coefficients $a, b$ and $d$ under these inequalities. More is said about this example in Section 4 below.

For the 'weak generic' delay kernel $d h(s)=k_{0}(s) d s=w e^{-w s} d s, w>0$, we see from the results in Section 2 that (3.4) fails to hold because $C_{1}(p)=w^{2} /\left(w^{2}+f^{2}\right)>$ $0, f=2 \pi / p$. Thus for this model the $p$-periodic solutions of Corollary 1 (again, for small $\varepsilon$ ) are associated with a coefficient $a<0$, i.e. an instantaneous autocatalytic response to density (or an Allee effect) in the growth rate of the species.

For the 'strong generic' delay kernel $d h(s)=k_{1}(s) d s=w^{2} s e^{-w s} d s, w>0$, however, the condition (3.4) is fulfilled when $2 \pi>w p$ since

$$
C_{1}(p)=w^{2}\left(w^{2}-f^{2}\right) /\left(w^{2}+f^{2}\right)^{2}, f=2 \pi / p .
$$

Since (H2) holds (see Example 2) Corollary 1 yields $p$-periodic solutions for coefficients $a>0, b>0$ for periods $p<2 \pi / w$.

Remark 5. Theorem 2 applies to the logistic equation (3.2) for any $m \geqslant 2$ and as a result $\varepsilon$ expansions of any desired order $m$ can be found for the periodic solutions $N(t)$ and the parameters $a, b$ and $d$ as described in Theorem 2 and its proof. An example of this is given in the next section.

Remark 6. An easy integration over the interval $0 \leqslant t \leqslant p$ of both sides of Equation (3.2) for a $p$-periodic solution shows that the average $[N]=p^{-1} \int_{0}^{p} N(t) d t$ equals the equilibrium $b /(a+d)$.

\section{The Lag-Logistic Model}

A model which has been frequently studied in the literature is the linear-response model (3.2) with $a=0$ (see for example $[6,7,9,10,15,16]$ and other references cited in these papers). This model is a delay version of the well-known logistic 
growth model and also arises in applications in fields other than population dynamics [9]. With our approach in this paper it is not clear that the results which we have derived apply to the case when $a=0$, since in Corollary $1 a$ is a function of $\varepsilon$. However, in Corollary 1 we have found 'branches' of solutions of the form $N(t, \varepsilon), a(\varepsilon), b(\varepsilon), d(\varepsilon)$ for small values of a parameter $\varepsilon$ which bifurcate from the values $e, \alpha_{1}, \alpha_{1}+\alpha_{2}, \alpha_{2}$ respectively and it is conceivable that for one or more of these branches (i.e. for one or more periods $p$ ) that $a(\varepsilon)=0$ for some value of $\varepsilon \neq 0$. In such an event the solution $N$ on this branch would solve, for this $\varepsilon$, the equation (3.2) with $a=0$ (and certain values of $b$ and $d$ ). It is of course possible to cause $\alpha_{1}=0$ and hence to make $a=0$ to lowest order in $\varepsilon$ (namely, by choosing a period $p$ for which $\left.C_{1}(p)=0\right)$, but since in general it is not true that $a(\varepsilon) \equiv 0$ the corresponding branch will have $a(\varepsilon) \neq 0$ for $\varepsilon \neq 0$.

One way to investigate the possibility that $a(\varepsilon)=0$, at least to lower order terms in $\varepsilon$, is to compute some lower order terms in the $\varepsilon$ expansions of $N, a, b$ and $d$ as given in Theorem 2 . We will do this in some detail for the case of a constant time lag of length $T>0$ in (3.2) which, after a time scaling, we assume without loss in generality is $T=1$. Thus, we take $h(s)=u_{1}(s)$ in (3.2) and consider

$$
\frac{1}{N(t)} \frac{d N(t)}{d t}=b-a N(t)-d N(t-1), \quad b \text { and } d>0 .
$$

We again assume (3.3) so that $e=1$ is the equilibrium. This equation has been studied in great detail in the case $a=0$ by Jones $[9,10]$ and others. We have already pointed out in Remark 4 that Corollary 1 applies when $\sin 2 \pi p^{-1} \neq 0$ and hence for such periods $p$ satisfying this condition we have the existence of non-constant $p$-periodic solutions for certain values of the parameters $a, b$ and $d$. We further assume

$$
\sin 2 \pi p^{-1}>0
$$

in which case we see from Corollary 1 that $d>0, b>0$, for small $\varepsilon$.

We apply Theorem 2 with $m=3$ (it applies to (4.1) for any $m \geqslant 2$ ) and conclude that (4.1) has solutions of the form

$$
\begin{aligned}
& N=1+\varepsilon y_{1}+\varepsilon^{2} y_{2}+\varepsilon^{3} y_{3}+\varepsilon^{3} z(\varepsilon) \quad \text { for } y_{2}, y_{3} \text { and } z \in P_{0} \\
& a=\alpha_{1}+\delta_{1} \varepsilon+\gamma_{1} \varepsilon^{2}+\varepsilon^{2} \beta_{1}(\varepsilon), \quad d=\alpha_{2}+\delta_{2} \varepsilon+\gamma_{2} \varepsilon^{2}+\varepsilon^{2} \beta_{2}(\varepsilon)
\end{aligned}
$$

and $b=a+d$.

The Equation (4.1) is autonomous in the sense that time translates of solutions are also solutions. As a result we loose no generality in choosing $y_{1}=\sin 2 \pi p^{-1} t$ in Theorems 1, 2 and Corollary 1 and hence in the expansions (4.3). If we substitute (4.3) into (4.1) and equate like powers of $\varepsilon$ we find that

$$
y_{2}^{\prime}=-\alpha_{1} y_{2}-\alpha_{2} y_{2}(t-1)-\delta_{1} y_{1}-\delta_{2} y_{2}(t-1)-\alpha_{1} y_{1}^{2}-\alpha_{2} y_{1}(t-1) y_{1}
$$


which is to be solved for $y_{2} \in P_{0}(p)$. Since $y_{1}^{2}$ and $y_{1}(t-1) y_{1}$ are easily shown to lie in $P_{0}(p)$ we see that (4.4) is solvable uniquely in $P_{0}(p)$ if and only if $\delta_{1}=\delta_{2}=0$. Then a straightforward Fourier analysis shows that

$$
\begin{array}{ll}
y_{2}=A \sin 4 \pi p^{-1} t+B \cos 4 \pi p^{-1} t \in P_{0}(p) \\
A=\pi \Omega p^{-1} /\left(\Omega^{2}+\Gamma^{2}\right), & B=-\pi \Gamma p^{-1} /\left(\Omega^{2}+\Gamma^{2}\right) \\
\Omega=\alpha_{1}+\alpha_{2} \cos 4 \pi p^{-1}, & \Gamma=4 \pi p^{-1}-\alpha_{2} \sin 4 \pi p^{-1}
\end{array}
$$

The equation for $y_{3} \in P_{0}(p)$ is then found to be

$$
\begin{aligned}
y_{3}^{\prime}= & -\alpha_{1} y_{3}-\alpha_{2} y_{3}(t-1)-\gamma_{1} y_{1}-\gamma_{2} y_{1}(t-1) \\
& -2 \alpha_{1} y_{1} y_{2}(t-1)-\alpha_{2} y_{2} y_{1}(t-1)
\end{aligned}
$$

the last five terms on the right hand side of which must be made to lie in $P_{0}(p)$ by an appropriate choice of $\gamma_{1}$ and $\gamma_{2}$. In order to find the first nonzero $0(\varepsilon)$ terms of the coefficients $a, b$ and $d$ these constants $\gamma_{1}$ and $\gamma_{2}$ must be computed, a task which is obviously quite involved. Thus, rather than compute exact formulas for $\gamma_{i}$ we will obtain approximations valid for periods $p$ near a critical value of interest, specifically, we will consider periods $p$ near $p=4$. The reason for this is as follows. Since we are interested in the case $a=0$ it is natural to consider the case when the lowest order term $\alpha_{1}=-2 \pi p^{-1} \cot 2 \pi p^{-1}$ in $a$ is small, i.e. when $\xi=\pi / 2-2 \pi p^{-1}=$ $\pi(p-4) / 2 p$ is small. Treating $\gamma_{1}$ and $\gamma_{2}$ as functions of $\xi$ we wish to compute these quantities only to order $0(\xi)$.

First of all $C_{1}(p)=\cos 2 \pi p^{-1}=\xi+0\left(\xi^{2}\right)$ and $S_{1}(p)=\sin 2 \pi p^{-1}=1-$ $(1 / 2) \xi^{2}+0\left(\xi^{4}\right)$ from which follows

$$
\begin{aligned}
& \alpha_{1}=-\pi \xi / 2+\xi^{2}+0\left(\xi^{3}\right), \quad \alpha_{2}=\pi / 2-\xi+\frac{\pi}{4} \xi^{2}+0\left(\xi^{3}\right), \\
& \Omega=-\pi / 2+0(\xi), \quad \Gamma=\pi+0(\xi)
\end{aligned}
$$

and hence

$$
A=-\frac{1}{10}+0(\xi), \quad B=-\frac{1}{3}+0(\xi)
$$

in $y_{2}(t)$. The two orthogonality conditions for (4.5) lead to the equations

$$
\begin{aligned}
\frac{1}{2} \gamma_{1} & +\left(\frac{1}{2} \cos 2 \pi p^{-1}\right) \gamma_{2}=\frac{\pi}{80}+0(\xi) \\
-\left(\frac{1}{2} \sin 2 \pi p^{-1}\right) \gamma_{2} & =-\frac{3 \pi}{80}+0(\xi)
\end{aligned}
$$

for $\gamma_{1}$ and $\gamma_{2}$, which yield finally

$$
\gamma_{1}=\frac{\pi}{40}+0(\xi), \quad \gamma_{2}=\frac{3 \pi}{40}+O(\xi)
$$


If we drop all terms of order $0\left(\xi^{p} \varepsilon^{q}\right)$ for $p+q \geqslant 3$ we obtain the approximations

$$
\begin{aligned}
& a \approx--\frac{\pi}{2} \xi+\xi^{2}+\frac{\pi}{40} \varepsilon^{2}, \quad d \approx \frac{\pi}{2}-\xi+\frac{\pi}{4} \xi^{2}+\frac{3 \pi}{40} \varepsilon^{2} \\
& b \approx \frac{\pi}{2}-\left(1+\frac{\pi}{2}\right) \xi+\left(1+\frac{\pi}{4}\right) \xi^{2}+\frac{\pi}{10} \varepsilon^{2} \\
& N(t) \approx 1+\varepsilon \sin 2 \pi p^{-1} t+\left(-\frac{1}{10} \sin 4 \pi p^{-1} t-\frac{1}{5} \cos 4 \pi p^{-1} t\right) \varepsilon^{2}
\end{aligned}
$$

where $\xi=\pi(p-4) / 2 p$ for the solutions of the lag-logistic model (4.1) for small $\varepsilon$ and for $p \approx 4$.

Returning now to the question of $a=0$, we choose $\varepsilon=\varepsilon_{0}$ so that the lower order approximation of $a$ given in (4.6) vanishes:

$$
\varepsilon_{2}^{0}=\xi\left(\frac{\pi}{2}-\xi\right) \frac{40}{\pi}=40 \pi(p-4) / p^{2} .
$$

Substitution of this $\varepsilon$ into (4.6) yields approximations for $N(t), b$ and $d$.

To summarize: given a period $p>4, p \approx 4$ there exists a p-periodic soluticn of

$$
\frac{1}{N(t)} \frac{d N(t)}{d t}=b(1-N(t-1))
$$

of the form (4.7) for $\varepsilon \approx \varepsilon_{0}$ given by (4.8) and for

$$
b \approx \frac{\pi}{2}+\left(\frac{3 \pi}{2}-1\right) \xi+\left(\frac{\pi}{4}-3\right) \xi^{2}, \quad \xi=\pi(p-4) / 2 p>0 .
$$

This result is consistent with those of Jones [9, 10] and others (e.g. Walther [15]), which assert the existence of a periodic solution for each $b>\pi / 2$. Note that in our result above $b>\pi / 2$ (for small $\varepsilon$ ) and that the solutions found are the only small amplitude periodic solutions (Remark 1).

Although Jones gives no formula for the period, his estimates $[9,10]$ imply $2<p<6$ which is again consistent with our result above.

In Figure 2 is drawn the bifurcation surface $S$ for (4.1) as described in a general setting in Figure 1 except that in Figure 2 we have used the original variables in (4.1) so that there appears a plot of the maximum (minimum) of the solution $N$ for $\varepsilon>0(\varepsilon<0)$ against $a$ and $b$. Here the equilibrium is $N=1$ (instead of 0 as in Figure 1). If $\xi \ll \varepsilon$ so that from (4.6)

$$
a \approx-\frac{\pi}{2} \xi+\frac{\pi}{40} \varepsilon^{2}, \quad b \approx \frac{\pi}{2}-\left(1+\frac{\pi}{2}\right) \xi+\frac{\pi}{10} \varepsilon^{2}
$$

then the base curve $B_{j}$ are approximated by the straight lines $b=4 a+\pi / 2+$ $(3 \pi / 2-1) \xi$ and the bifurcation curve $B$ is approximated by the straight line $b=$ $(1+2 / \pi) a+\pi / 2$. Solutions of (4.9) are obtained by considering bifurcation 
along the curve $\Lambda$ given by $a=0$ (i.e. by slicing the bifurcation surface $S$ by the coordinate plane $a=0$ ) which results in a typical 'right handed' bifurcation curve $C$ of Hopf-type (or period varying type) for (4.9).

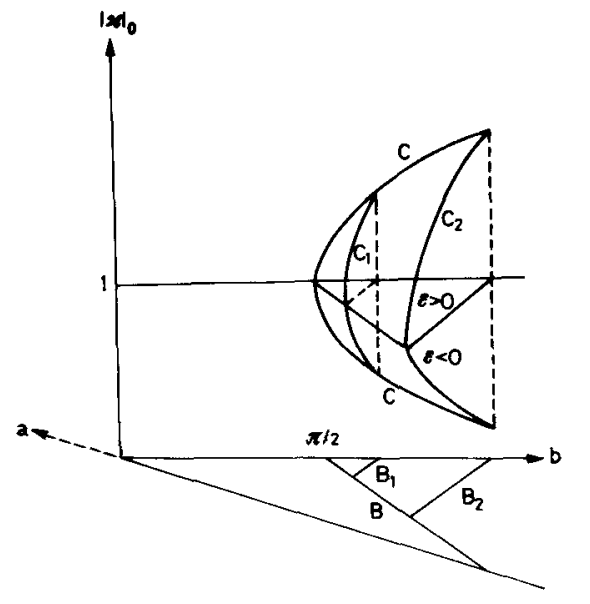

Figure 2

\section{References}

1. Cushing, J. M.: Periodic solutions of Volterra's population equation with hereditary effects, SIAM J. Appl. Math. 31, 251-261 (1976)

2. Cushing, J. M.: Errata to Periodic solutions of Volterra's population equation with hereditary effects, SIAM Appl. Math. 32, 895 (1977)

3. Cushing, J. M.: Time delays in single species growth models, J. Math. Biology 4, 257-264 (1977)

4. Cushing, J. M.: Bifurcation of periodic solutions of integrodifferential systems with application to time delay models in population dynamics, SIAM J. Appl. Math. 33, 640-654 (1977)

5. Cushing, J. M.: Integrodifferential Equations and Delay Models in Population Dynamics, Lec. Notes in Biomath. 20, Berlin, Heidelberg, New York: Springer, 1977

6. Dunkel, G. M.: Some mathematical models for population growth with lags, thesis, University of Maryland, College Park, Md., 1968

7. Dunkel, G. M.: Single species model for population growth depending on past history, pp. 92-99, Lec. Notes in Math. 60, Berlin, Heidelberg, New York: Springer, 1968.

8. Grafton, R. B.: A periodicity theorem for autonomous functional differential equations, J. Diff. Eqs. 6, 87-109 (1969)

9. Jones, G. S.: The existence of periodic solutions of $f^{\prime}(x)=-\alpha f(x-1)\{1+f(x)\}$, J. Math. Anal. Appl. 5, 435-450 (1962)

10. Jones, G. S.: On the nonlinear differential-difference equation $f^{\prime}(x)=-\alpha f(x-1)\{1+$ $f(x)$ \}, J. Math. Anal. Appl. 4, No. 3, 440-469 (1962)

11. Kakutani, S., Markus, L.: On the nonlinear difference-differential equation $y^{\prime}(t)=$ $(A-B y(t-\tau)) y(t)$, in Contributions to the Theory of Nonlinear Oscillations, pp. 1-18, Princeton, New Jersey: Univ. Press, 1958

12. May, R. M., Conway, G. R., Hassell, M. P., Southwood, T. R. E.: Time delays, densitydependence and single species oscillations, J. Anim. Ecol. 43, No. 3, 747-770 (1974)

13. Miller, R. K.: On Volterra's population equation, SIAM J. Appl. Math. 14, No. 3, 446-452 (1966) 
14. Nussbaum, R. D.: Periodic solutions of some nonlinear, autonomous functional differential equations II, J. Diff. Eq. 14, 360-394 (1973)

15. Walther, H. O.: Existence of a non-constant periodic solution of a nonlinear autonomous functional differential equation representing the growth of a single species population $\mathrm{J}$. Math. Biology 1, 227-240 (1975)

16. Wright, E. M.: A nonlinear difference-differential equation, J. Reine Angew. Math. 194, 66-87 (1955)

Received November 11, 1977/Revised January 31, 1978 